\title{
TILTING INVARIANCE OF THE AUSLANDER-REITEN CONJECTURE
}

\author{
JIAQUN WEI
}

\begin{abstract}
Let $R$ be an artin algebra and $T$ be a tilting or cotilting $R$-module with $S=\operatorname{End}_{R} T$. We show that $R$ satisfies the Auslander-Reiten conjecture if and only if so does $S$.
\end{abstract}

\section{Introduction}

Throughout this paper, we consider artin algebras and finitely generated left modules over them. Let $R$ be an artin algebra, we denote by $\bmod R$ the category of finitely generated left $R$-modules. For an $X \in \bmod R$, we denote by $\operatorname{pd}_{R} X\left(\operatorname{resp} ., \operatorname{id}_{R} X\right)$ the projective (resp., injective) dimension of $X$.

In studying the Nakayama conjecture, Auslander and Reiten [2] proposed the following conjecture, which is also listed as the 10th conjecture in the book [4].

Auslander-Reiten Conjecture: Let $R$ be an artin algebra and $X$ be an $R$ module. If $\operatorname{Ext}_{R}^{i}(X, X \oplus R)=0$ for all $i \geq 1$, then $X$ is projective.

Auslander and Reiten [2] proved the conjecture over artin algebras such that every module $M$ has an ultimately closed projective resolution, that is, there is some syzygy $N$ of $M$ such that all indecomposable direct summands of $N$ already appear in earlier syzygies. This includes algebras of finite representation type, algebras with radical square zero and all torsionless-finite algebras. Hoshino [9] proved if $R$ is a self-injective artinian local ring with radical cube zero, then $\operatorname{Ext}_{R}^{1}(M, M)=0$ implies that $M$ is free.

We note that the Auslander-Reiten Conjecture actually makes sense for any ring. In fact, there are already some results in the study of the Auslander-Reiten Conjecture for commutative algebras, see for instance $[1,5,11,12]$ etc.. Recently, Christensen and Holm proved that every left noetherian ring satisfy the Auslander-Reiten Conjecture if it satisfies the Auslander's condition on vanishing of cohomology [6]. Such rings contain group algebras of finite groups and artin algebras such that every module $M$ has an ultimately closed projective resolution $[6,16]$.

The Auslander-Reiten Conjecture is related to the Finitistic Dimension Conjecture which reads as follows.

Finitistic Dimension Conjecture: Let $R$ be an artin algebra. Then fdim $R=$

Received by the editors March 28, 2009.

2000 Mathematics Subject Classification. Primary 16E10; Secondary 16E30.

Key words and phrases. Auslander-Reiten conjecture, tilting invariance.

Supported by the National Natural Science Foundation of China (Grant Nos. 10601024 and 10971099. 
$\sup \left\{\operatorname{pd}_{R} X \mid \operatorname{pd}_{R} X<\infty\right\}$ is finite.

Indeed, if the Finitistic Dimension Conjecture holds for all artin algebras then the Auslander-Reiten Conjecture holds for all artin algebras. However, for an artin algebra satisfying the Finitistic Dimension Conjecture, we don't know if it also satisfies the Auslander-Reiten Conjecture. For instance, it is still a question if the AuslanderReiten Conjecture holds for all self-injective artin algebras and in this case, it is just the Tachikawa conjecture [15]. However, there is a counterexample over QF-rings [14].

Our aim in this paper is to show that the Auslander-Reiten conjecture is in fact a tilting invariance. More precisely, we prove the following result.

Main Theorem Let $R$ be an artin algebra and $T \in \bmod R$ be a tilting module with $S=\operatorname{End}_{R} T$. Then $R$ satisfies the Auslander-Reiten conjecture if and only if so does $S$.

Similar results for Finitistic Dimension Conjecture had been proved by Happel [7] using the techniques of derived categories. More recently, it was proved that the Finitistic Dimension Conjecture is stable under derived equivalences. It would be interesting to consider whether the Auslander-Reiten conjecture is also stable under derived equivalences.

\section{Preliminaries}

Let $R$ be an artin algebra and $M \in \bmod R$. We denote by $R^{o}$ the opposite algebra and an $R^{o}$-module $M$ means the right $R$-module $M_{R}$.

Let $\mathcal{C}$ be a subcategory of $\bmod R$, we denote by $\mathcal{C}$ the category of all $R$-modules $M$ such that there is an exact sequence $0 \rightarrow M \rightarrow C_{0} \rightarrow \cdots \rightarrow C_{m} \rightarrow 0$ for some integer $m$ with each $C_{i} \in \mathcal{C}$. Let $M \in \mathcal{C}$, we denote by $\operatorname{codim}_{\mathcal{C}}(M)$ the minimal non-negative integer $m$ such that there is an exact sequence $0 \rightarrow M \rightarrow C_{0} \rightarrow \cdots \rightarrow C_{m} \rightarrow 0$ with each $C_{i} \in \mathcal{C}$. We also denote by $(\mathcal{C})_{n}$ the category of all $M \in \mathcal{C}$ with $\operatorname{codim}_{\mathcal{C}}(M) \leq n$. Dually, the notion $\widehat{\mathcal{C}}$ denotes the category of all $R$-modules $M$ such that there is an exact sequence $0 \rightarrow C_{m} \rightarrow \cdots \rightarrow C_{0} \rightarrow M \rightarrow 0$ for some integer $m$ with each $C_{i} \in \mathcal{C}$, and the notion $\operatorname{dim}_{\mathcal{C}}(M)$ denotes the minimal non-negative integer $m$ such that there is an exact sequence $0 \rightarrow C_{m} \rightarrow \cdots \rightarrow C_{0} \rightarrow M \rightarrow 0$ with each $C_{i} \in \mathcal{C}$. Similarly, the notion $(\widehat{\mathcal{C}})_{n}$ is the category of all $M \in \widehat{\mathcal{C}}$ with $\operatorname{dim}_{\mathcal{C}}(M) \leq n$.

Let $M \in \bmod R$. We denote by $\operatorname{add}_{R} M$ the category of modules isomorphic to direct summands of finite direct sums of $M$. The notion $M^{\perp}$ denotes the category of all modules $N \in \bmod R$ such that $\operatorname{Ext}_{R}^{i \geq 1}(M, N)=0$. Dually, the notion ${ }^{\perp} M$ denotes the category of all modules $N$ such that $\operatorname{Ext}_{R}^{i \geq 1}(N, M)=0$.

We denote by $\mathbf{D}$ the usual duality functor between $\bmod R$ and $\bmod R^{o}$. For an $M \in \bmod R$ and a positive integer $t$, the notion $\Omega_{R}^{t} M$ denote the $t$-th syzygy of $M$.

We recall now some necessary basic tilting theory. The readers are suggested to refer to $[3,8,13]$ for more details.

Let $R$ be an artin algebra and $n$ a non-negative integer. Recall that $T \in \bmod R$ is called a tilting module of projective dimension at most $n$ if it satisfies the following three conditions:

(T1) $\operatorname{pd} T \leq n$, i.e., there is an exact sequence $0 \rightarrow R_{n} \rightarrow \cdots \rightarrow R_{0} \rightarrow T \rightarrow 0$ with each $R_{i}$ projective.

(T2) $\operatorname{Ext}_{R}^{i}(T, T)=0$ for all $i>0$, and 
(T3) there is an exact sequence $0 \rightarrow R \rightarrow T_{0} \rightarrow \cdots \rightarrow T_{n} \rightarrow 0$ for some $n$, where each $T_{i} \in \operatorname{add} T$.

The notion of tilting modules is left-right symmetric in the sense that if ${ }_{R} T$ is a tilting module of projective dimension at most $n$, then $T_{S}$, where $S=\operatorname{End}_{R} T$, is also a tilting module of projective dimension at most $n$.

Dually, $C \in \bmod R$ is a cotilting module of injective dimension at most $n$ if it satisfies

(C1) $\operatorname{id} T \leq n$,

(C2) $\operatorname{Ext}_{R}^{i}(T, T)=0$ for all $i>0$, and

(C3) there is an exact sequence $0 \rightarrow C_{n} \rightarrow \cdots \rightarrow C_{0} \rightarrow \mathbf{D}\left(R_{R}\right) \rightarrow 0$ with each $C_{i} \in \operatorname{add}_{R} C$.

Note that $T \in \bmod R$ is a tilting module of projective dimension at most $n$ if and only if $\mathbf{D}\left({ }_{R} T\right) \in \bmod R^{o}$ is a cotilting module of injective dimension at most $n$.

Lemma 2.1. Let $R$ be an artin algebra and $T \in \bmod R$ be a tilting module of projective dimension at most $n$. Then for any $M \in \bmod R$, there is an exact sequence $0 \rightarrow M \rightarrow$ $U_{M} \rightarrow V_{M} \rightarrow 0$ with $U_{M} \in T^{\perp}$ and $V_{M} \in\left(\operatorname{add}_{R} T\right)_{n-1}$. In particular, $V_{M}$ has the projective dimension at most $n$.

Proof. The claimed exact sequence exists by for instance [3, Section 5].

The following is the well-known Brenner-Butler Theorem in the tilting theory, see for instance $[8,13]$.

Lemma 2.2. Let $R$ be an artin algebra and $T \in \bmod R$ be a tilting module with $S=\operatorname{End}_{R} T$. Denote $C=\mathbf{D}\left(T_{S}\right)$. Then there is an equivalence between $T^{\perp}$ and ${ }^{\perp} C$, given by the functor $\operatorname{Hom}_{R}(T,-)$. Moreover, for any $U, W \in T^{\perp}$ and any $i \geq 0$, we have that $\operatorname{Ext}_{R}^{i}(U, W) \simeq \operatorname{Ext}_{S}^{i}\left(\operatorname{Hom}_{R}(T, U), \operatorname{Hom}_{R}(T, W)\right)$ canonically.

\section{The proof of Main Theorem}

Throughout this section, we fix an artin algebra $R$ and a tilting $R$-module $T$ with $S=\operatorname{End}_{R} T$. We set $n=\operatorname{pd}_{R} T$.

To prove the Main Theorem, we need some lemmas.

Lemma 3.1. Assume that $M \in \bmod R$ satisfies that $M \in{ }^{\perp}(M \oplus R)$. Then $\Omega_{R}^{n} M \in$ ${ }^{\perp}\left(\Omega_{R}^{n} M \oplus R\right)$.

Proof. Consider the exact sequence $0 \rightarrow \Omega^{n} M \rightarrow R_{n-1} \rightarrow \cdots \rightarrow R_{0} \rightarrow M \rightarrow 0$ with each $R_{i}$ projective. By applying functors $\operatorname{Hom}_{R}(-, R), \operatorname{Hom}_{R}\left(-, \Omega^{n} M\right)$ and $\operatorname{Hom}_{R}(M,-)$ in turn, we obtain for all $i \geq 1$ that firstly

$$
\operatorname{Ext}_{R}^{i}\left(\Omega^{n} M, R\right) \simeq \operatorname{Ext}_{R}^{i+n}(M, R)=0,
$$

secondly

and lastly

$$
\operatorname{Ext}_{R}^{i}\left(\Omega^{n} M, \Omega^{n} M\right) \simeq \operatorname{Ext}_{R}^{i+n}\left(M, \Omega^{n} M\right)
$$

$$
\operatorname{Ext}_{R}^{i+n}\left(M, \Omega^{n} M\right) \simeq \operatorname{Ext}_{R}^{i}(M, M)=0,
$$

by the dimension shift and the assumption. Hence the conclusion follows.

Lemma 3.2. Assume that $M \in{ }^{\perp} T$. Then $\Omega_{R}^{n} M \in{ }^{\perp} R$. 
Proof. Consider the exact sequence $0 \rightarrow R \rightarrow T_{0} \rightarrow \cdots \rightarrow T_{n} \rightarrow 0$ in the definition of tilting modules. Applying the functor $\operatorname{Hom}_{R}(M,-)$, we obtain that $\operatorname{Ext}_{R}^{i+n}(M, R) \simeq$ $\operatorname{Ext}_{R}^{i}\left(M, T_{n}\right)=0$ for all $i \geq 1$ by the dimension shift, since $M \in{ }^{\perp} T$. It follows that $\operatorname{Ext}_{R}^{i}\left(\Omega_{R}^{n} M, R\right) \simeq \operatorname{Ext}_{R}^{i+n}(M, R)=0$ for all $i \geq 1$, i.e., $\Omega_{R}^{n} M \in{ }^{\perp} R$.

The following lemma is important for the proof.

Lemma 3.3. For any $M \in \bmod R$, there is an exact sequence

$$
0 \rightarrow M \rightarrow V \rightarrow U \rightarrow 0
$$

such that $U \in \operatorname{add}_{R} T$ and $V$ satisfies an exact sequence

with each $T_{i} \in \operatorname{add}_{R} T$.

$$
0 \rightarrow \Omega_{R}^{n} M \rightarrow T_{n-1} \rightarrow \cdots \rightarrow T_{0} \rightarrow V \rightarrow 0
$$

Proof. Clearly we have the exact sequence $0 \rightarrow \Omega^{n} M \rightarrow R_{n-1} \rightarrow \cdots \rightarrow R_{0} \rightarrow M \rightarrow 0$ with each $R_{i}$ projective. Since $R \in\left(\operatorname{add}_{R} T\right)_{n}$, the conclusion then follows from [17, Lemma 2.3].

Lemma 3.4. Assume that $M \in{ }^{\perp}(T \oplus M) \bigcap T^{\perp}$. Then $\Omega_{R}^{n} M \in{ }^{\perp} \Omega_{R}^{n} M$.

Proof. We consider the two exact sequences in Lemma 3.3. Since $\operatorname{pd}_{R} T \leq n$ and $\operatorname{Ext}_{R}^{i}(T, T)=0$ for all $i \geq 1$, we easily obtain that $V \in T^{\perp}$ from the exact sequence ( ). It follows that $U \in T^{\perp}$ from the exact sequence $(\dagger)$, since $M \in T^{\perp}$ too. Hence we obtain that $U \in T^{\perp} \bigcap \operatorname{add}_{R} T=\operatorname{add}_{R} T$. It turns out that the sequence ( $\dagger$ ) splits, and consequently $V \simeq M \oplus U \in \operatorname{add}_{R}(T \oplus M)$. Since $M \in{ }^{\perp}(T \oplus M)$ by assumption, $M \in{ }^{\perp} V$ too. Hence, applying the functor $\operatorname{Hom}_{R}(M,-)$ to the exact sequence ( $\left.\ddagger\right)$, we obtain that $\operatorname{Ext}_{R}^{i+n}\left(M, \Omega_{R}^{n} M\right) \simeq \operatorname{Ext}_{R}^{i}(M, V)=0$ for all $i \geq 1$ by the dimension shift. It follows that $\operatorname{Ext}_{R}^{i}\left(\Omega_{R}^{n} M, \Omega_{R}^{n} M\right) \simeq \operatorname{Ext}_{R}^{i+n}\left(M, \Omega_{R}^{n} M\right)=0$ for all $i \geq 1$, i.e., $\Omega_{R}^{n} M \in{ }^{\perp} \Omega_{R}^{n} M$.

Lemma 3.5. Assume that $M \in^{\perp}(T \oplus M) \cap T^{\perp}$. If $R$ satisfies the Auslander-Reiten conjecture, then $M \in \operatorname{add}_{R} T$.

Proof. Since $M \in{ }^{\perp}(T \oplus M) \bigcap T^{\perp}$, we obtain that $\Omega_{R}^{n} M \in{ }^{\perp}\left(\Omega_{R}^{n} M \oplus R\right)$ by Lemmas 3.2 and 3.4. If $R$ satisfies the Auslander-Reiten conjecture, then $\Omega_{R}^{n} M$ must be projective. It follows that $\operatorname{pd}_{R} M<\infty$. Combining with the assumption $M \in T^{\perp}$, we have that $M \in \widehat{\operatorname{add}_{R} T}$. Combining with the assumption $M \in{ }^{\perp} T$, we easily obtain that $M \in \operatorname{add}_{R} T$.

We can now prove the one-part of the Main Theorem.

Proposition 3.6. If $R$ satisfies the Auslander-Reiten conjecture, then so does $S$.

Proof. Take any $N \in \bmod S$ such that $N \in{ }^{\perp}(N \oplus S)$. Then $\Omega_{S}^{n} N \in{ }^{\perp}\left(\Omega_{S}^{n} N \oplus S\right)$ by Lemma 3.1. Note that $\Omega_{S}^{n} N \in{ }^{\perp} \mathbf{D}\left(T_{S}\right)$, so $\Omega_{S}^{n} N=\operatorname{Hom}_{R}(T, M)$ for some $M \in T^{\perp}$ by the tilting equivalence in Lemma 2.2. Since $S=\operatorname{Hom}_{R}(T, T)$ and $M \oplus T \in T^{\perp}$, we obtain that $\operatorname{Ext}_{R}^{i}(M, M \oplus T) \simeq \operatorname{Ext}_{S}^{i}\left(\operatorname{Hom}_{R}(T, M), \operatorname{Hom}_{R}(T, M) \oplus \operatorname{Hom}_{R}(T, T)\right) \simeq$ $\operatorname{Ext}_{S}^{i}\left(\Omega_{S}^{n} N, \Omega_{S}^{n} N \oplus S\right)=0$ for all $i \geq 1$ by assumption and Lemma 2.2 again. Hence we have that $M \in{ }^{\perp}(T \oplus M) \cap T^{\perp}$. Since $R$ satisfies the Auslander-Reiten conjecture, we obtain from Lemma 3.5 that $M \in \operatorname{add}_{R} T$. It follows that $\Omega_{S}^{n} N\left(=\operatorname{Hom}_{R}(T, M)\right)$ is projective. Consequently, $\operatorname{pd}_{S} N<\infty$. Since $N \in{ }^{\perp} S$ too, it is easy to see that $N$ is projective. It follows that $S$ satisfies the Auslander-Reiten conjecture. 


\section{Proof of the Main Theorem:}

By the previous proposition, we need only to show that if $S$ satisfies the AuslanderReiten conjecture, then so does $R$. To this end, let us take any $M \in \bmod R$ such that $M \in{ }^{\perp}(M \oplus R)$. Then we need to show that $M$ is projective.

By Lemma 2.1, there is exact sequence

$$
0 \rightarrow M \rightarrow U_{M} \rightarrow V_{M} \rightarrow 0
$$

with $U_{M} \in T^{\perp}$ and $V_{M} \in\left(\operatorname{add}_{R} T\right)_{n-1}$. Note that for any $N \in \bmod R$ with $\operatorname{pd}_{R} N<$ $\infty$, we have that $\operatorname{Ext}_{R}^{i}(M, N)=0$ for all $i \geq 1$ since $M \in{ }^{\perp} R$. It follows that $M \in{ }^{\perp} T$ and $M \in{ }^{\perp} V_{M}$. Since clearly $V_{M} \in{ }^{\perp} T$ too, we obtain that $U_{M} \in{ }^{\perp} T$ from the sequence $(\sharp)$. By assumption, $\operatorname{Ext}_{R}^{i}(M, M)=0$ for all $i \geq 1$. It follows that $\operatorname{Ext}_{R}^{i}\left(M, U_{M}\right)=0$ for all $i \geq 1$ by applying the functor $\operatorname{Hom}_{R}(M,-)$ to the sequence $(\sharp)$. Note also that $\operatorname{Ext}_{R}^{i}\left(V_{M}, U_{M}\right)=0$ for all $i \geq 1$ since $U_{M} \in T^{\perp}$ and $V_{M} \in \operatorname{add}_{R} T$, so applying the functor $\operatorname{Hom}_{R}\left(-, U_{M}\right)$ to the exact sequence $(\sharp)$, we further obtain that $\operatorname{Ext}_{R}^{i}\left(U_{M}, U_{M}\right)=0$ for all $i \geq 1$. It amounts to that $U_{M} \in{ }^{\perp}\left(T \oplus U_{M}\right) \cap T^{\perp}$.

Denote $N=\operatorname{Hom}_{R}\left(T, U_{M}\right)$. Then by Lemma 2.2 and the above arguments, we obtain that $\operatorname{Ext}_{S}^{i}(N, N \oplus S)=\operatorname{Ext}_{S}^{i}\left(\operatorname{Hom}_{R}\left(T, U_{M}\right), \operatorname{Hom}_{R}\left(T, U_{M}\right) \oplus \operatorname{Hom}_{R}(T, T)\right) \simeq$ $\operatorname{Ext}_{R}^{i}\left(U_{M}, U_{M} \oplus T\right)=0$ for all $i \geq 1$. Hence if $S$ satisfies the Auslander-Reiten conjecture, then $N$ is projective. Consequently $U_{M} \in \operatorname{add}_{R} T$ by the tilting equivalence in Lemma 2.2. Thus from the exact sequence $(\sharp)$ we obtain that $M \in \operatorname{add}_{R} T$. It follows that $\operatorname{pd}_{R} M<\infty$, and hence $M$ is projective. Thus $R$ satisfies the Auslander-Reiten conjecture.

Corollary 3.7. Let $R$ be an artin algebra and $T \in \bmod R$ with $S=\operatorname{End}_{R} T$.

(1) If $T$ is a tilting module, then $R^{o}$ satisfies the Auslander-Reiten conjecture if and only if so does $S^{\circ}$.

(2) If $T$ is a cotilting module, then $R$ (resp., $\left.R^{o}\right)$ satisfies the Auslander-Reiten conjecture if and only if so does $S\left(\right.$ resp., $\left.S^{\circ}\right)$.

Proof. (1) Note that $T$ is also a tilting $S^{o}$-module with $R^{o} \simeq \operatorname{End}_{S^{o}} T$, so the conclusion follows from the Main Theorem.

(2) Denote $C=\mathbf{D}\left(T_{S}\right)$. Then $C$ is a tilting $S$-module. Now the conclusion follows from the Main Theorem and the first part.

Let $A, B$ be two artin algebras. We say that $A$ is tilting-cotilting equivalent to $B$, provided that there are some artin algebras $A_{i}, 0 \leq i \leq n$, and some tilting or cotilting $A_{i}$-modules $T_{i}$ such that $A=A_{0}, B=A_{n}$, and $A_{i+1} \simeq \operatorname{End}_{A_{i}} T_{i}$ for $0 \leq i \leq n-1$. Clearly the tilting-cotilting equivalence is a kind of derived equivalences. Results in this paper show that if two artin algebras $A$ and $B$ are tilting-cotilting equivalent, then $A$ satisfies the Auslander-Reiten conjecture if and only if so does $B$.

\section{Acknowledgements}

The author also thanks Professor Steffen König for his support through the network "Representation theory of algebras and algebraic Lie theory" funded by RheinenergieStiftung. 


\section{References}

[1] M. Auslander, S. Ding and $\varnothing$. Solberg, Liftings and weak liftings of modules, J. Algebra. 156 (1993), 273-317.

[2] M. Auslander and I. Reiten, On a generalized version of the Nakayama Conjecture, Proc. AMS. $\mathbf{5 2}$ (1975), 69-74.

[3] M. Auslander and I. Reiten, Applications of contravariantly finite subcategories, Adv. Math. 86 (1991), no.1, 111-152.

[4] M. Auslander, I. Reiten and S. O. Smalø, Representation theory of Artin algebras. Cambridge Univ. Press (1995).

[5] L.L. Avramov and R.-O. Buchweitz, Support varieties and cohomology over complete intersections, Invent. Math. 142 (2000), 285-318.

[6] L.W. Christensen and H. Holm, Algebras that satisfy Auslander's condition on vanishing of cohomology, Math. Zeit., to appear.

[7] D. Happel, Reduction techniques for homological conjectures, Tsukuba J.Math., 17 (1993), no. 1, 115-130.

[8] D. Happel, Triangulated categories in the representation theory of finite dimension algebras, London Matn. Soc. Lect. Notes Ser. 119, Cambridge (1988).

[9] M. Hoshino, Modules without self-extensions and Nakayama's conjecture, Arch. Math. 43 (1984), 494-500.

[10] C. Huneke and D.A. Jorgensen, Symmetry in the vanishing of Ext over Gorenstein rings, Math. Scand 93 (2003), no. 8, 161-184.

[11] C. Huneke and G.J. Leuschke, On a conjecture of Auslander and Reiten, J. Algebra 275 (2004), 781-790.

[12] C. Huneke, L.M. Sega and A.N. Vraciu, Vanishing of Ext and Tor over some Cohen-Macaulay local rings, Illinois J. Math. 48 (2004), 295-317.

[13] Y. Miyashita, Tilting modules of finite projective dimension, Math. Zeit. 193 (1986), 113-146.

[14] R. Schulz, A non-projective module without self-extensions, Arch. Math. 62 (1994), 497-500.

[15] H. Tahcikawa, Quasi-Frobenius Rings and Generalizations, Lect. Note Math. 351, 1973.

[16] J. Wei, A note on Auslander bounds, arXiv: 0802.1085v2.

[17] J. Wei and C. Xi, A characterization of tilting pairs, J. Algebra 317 (2007), 376-391.

Department of Mathematics, Nanjing Normal University, Nanjing 210097, P.R.China

E-mail address: weijiaqun@njnu.edu.cn

Current address: Mathematisches Institut, Universitaet Zu Koeln, Weyertal 86-90, D-50931 Koeln, Germany 